\title{
Sodium-glucose cotransporter 2 inhibitors (SGLT2i) and cardiac arrhythmias: a systematic review and meta-analysis
}

Hang-Long Li ${ }^{1,6}$, Gregory Y. H. Lip ${ }^{2,3}$, Qi Feng ${ }^{4}$, Yue Fei ${ }^{5}$, Yi-Kei Tse ${ }^{1}$, Mei-zhen Wu ${ }^{1,6}$, Qing-wen Ren ${ }^{1,6}$, Hung-Fat Tse ${ }^{1,6}$, Bernard-M. Y. Cheung ${ }^{5}$ and Kai-Hang Yiu ${ }^{1,6^{*}}$ (])

\begin{abstract}
Background: Cardiac arrhythmias are associated with poorer outcomes in patients with heart failure (HF), diabetes mellitus (DM), and chronic kidney disease (CKD). Previous studies have shown inconsistent conclusions regarding the association between sodium-glucose cotransporter 2 inhibitors (SGLT2i) and the risk of developing arrhythmias. This study aims to investigate the association of SGLT2i treatment with arrhythmia outcomes in clinical trials of patients with HF, DM, or CKD.
\end{abstract}

Methods: MEDLINE, EMBASE, and ClinicalTrials.gov were searched from inception up to 27 August 2020. Randomized controlled trials that randomized patients with DM, CKD, or HF to SGLT2i or placebo were included. The outcomes of interest include atrial fibrillation (AF), embolic stroke, atrial flutter (AFL), AF/AFL, ventricular tachycardia (VT), and cardiac arrest. Relative risks (RRs) and 95\% confidence intervals (Cl) were pooled using a random-effects model.

Results: Out of 4,532 citations, 22 trials with altogether 52,115 patients were included (mean age 63.2 years; 33,747 [64.8\%] of participants were men). SGLT2i were associated with a lower risk of AF (RR 0.82, 95\% Cl 0.70-0.96), embolic stroke (RR 0.32, 95\% Cl 0.12-0.85), AF/AFL (RR 0.82, 95\% Cl 0.71-0.95), and VT (RR 0.73, 95\% Cl 0.53-0.99), while the risk reductions in AFL (RR 0.83, 95\% Cl 0.58-1.17) and cardiac arrest (RR $0.83,95 \% \mathrm{Cl} 0.61-1.14$ ) did not reach statistical significance. The associations appeared to be consistent across different baseline conditions (DM vs CKD vs HF; atherosclerotic cardiovascular disease [ASCVD] vs no ASCVD) and the SGLT2i used.

Conclusions: SGLT2i reduced the risk of cardiac arrhythmias. Our study provides further evidence for recommending the use of SGLT2i in patients with DM, CKD, and HF. Further research is needed to fully elucidate the mechanism by which SGLT2i protect against arrhythmias.

Keywords: SGLT2 inhibitors, Arrhythmia, Atrial fibrillation

\section{Background}

Diabetes mellitus (DM) and related comorbidities including heart failure (HF), obesity, hypertension, and chronic kidney disease (CKD) are closely linked to atrial

\section{*Correspondence: khkyiu@hku.hk}

1 Division of Cardiology, Department of Medicine, The University of Hong Kong, Queen Mary Hospital, Room 1929B/K1931, Block K, Hong Kong,

China

Full list of author information is available at the end of the article fibrillation (AF) [1-5]. These conditions are associated with myocardial fibrosis and remodeling, neurohormonal activation, autonomic dysfunction, and electrical remodeling, predisposing to the development of AF and cardiac arrhythmias [2-6]. As AF and dysrhythmias are associated with a higher risk of adverse cardiovascular events and death, it is important to reduce the risk of cardiac arrhythmias in patients with HF, DM, and CKD [2, 4, 7-12]. 
Sodium-glucose cotransporter 2 inhibitors (SGLT2i) are antidiabetic medications which act by inhibiting the reabsorption of sodium and glucose in the proximal tubules of the kidney [13]. Commonly used SGLT2i include canagliflozin, dapagliflozin, and empagliflozin [13]. The cardioprotective effects of SGLT2i have been increasingly recognized in recent years: studies have shown that SGLT2i protected against atherosclerotic cardiovascular disease (ASCVD) and reduced HF hospitalization [13-15]. Furthermore, studies have shown that SGLT2i promoted weight loss and lowered blood pressure $[13,16]$. In view of the wide spectrum of cardiovascular benefits, it has been hypothesized that SGLT2i may reduce the risk of AF and cardiac arrhythmias [17].

However, the associations between SGLT2i and AF remained inconsistent across previous studies. A recent secondary analysis of the DECLARE-TIMI 58 trial (Dapagliflozin Effect on Cardiovascular Events-Thrombolysis in Myocardial Infarction 58) found that dapagliflozin reduced the risk of AF and atrial flutter (AFL) by $19 \%$ in susceptible patients with DM, compared to placebo [17]. The reduction in AF/AFL events was consistent regardless of the presence of ASCVD, HF, and AF at baseline. However, one previous meta-analysis did not identify a significant association between SGLT2i and AF [18]. In the EMPA-REG OUTCOME trial (Empagliflozin Cardiovascular Outcome Event Trial in Type 2 Diabetes Mellitus Patients), the empagliflozin arm had a higher incidence of new-onset AF (2.3\%) compared to the placebo arm (1.6\%), though it was not an adjudicated outcome of the trial [19]. Recent real-world studies have also shown inconsistent conclusions: while SGLT2i were associated with a lower incidence of new-onset arrhythmias and AF, [20,21] the CVD-REAL Nordic study [22] showed neutral association. Other types of arrhythmias, such as ventricular tachycardia (VT), as well as related conditions such as cardiac arrest, have been less well studied. Hence, the association between SGLT2i and arrhythmia outcomes remains uncertain.

Therefore, the objective of this systematic review and meta-analysis was to evaluate the effects of SGLT2i on common arrhythmia outcomes (AF, AFL, VT, and cardiac arrest) and related complications (embolic stroke) in patients with DM, CKD, and HF.

\section{Methods}

This systematic review and meta-analysis was conducted and reported according to the Cochrane Handbook (Version 5.1.0) [23] and the PRISMA statement [24]. The PRISMA checklist is shown in Additional file 1: Table S1.

\section{Data sources and searches}

Ovid MEDLINE, Ovid EMBASE, and ClinicalTrials.gov were searched for eligible studies through 27 August 2020. The search strategy is shown in Additional file 1: Table S2. Reviews articles and expert consensus statements were also manually searched for eligible studies.

\section{Study selection}

We included RCTs that compared SGLT2i with placebo in adult patients ( $\geq 18$ years) with type $2 \mathrm{DM}$, CKD, or $\mathrm{HF}$ and reported outcomes of interest as serious adverse events (SAEs). In order to ascertain the true anti-arrhythmic effects of SGLT2i, trials that randomized patients to combination therapy were excluded, and placebo was selected as a comparator. There were no restrictions on follow-up duration or the language of publication. The outcomes of interest include AF, embolic stroke, AFL, $\mathrm{AF} / \mathrm{AFL}, \mathrm{VT}$, and cardiac arrest. Titles and abstracts were first screened to assess their potential eligibility, followed by full-text examination to determine final eligibility.

\section{Data extraction and quality assessment}

The following information was extracted using a prespecified data extraction form: bibliographic information (First author, year of publication), study information (trial name, ClinicalTrials.gov unique identifier, country, sample size), patients characteristics (age, proportion of male patients, baseline conditions and comorbidities (DM, $\mathrm{CKD}, \mathrm{HF}, \mathrm{AF}$ )), treatment information (regimen, dose, duration), and outcome data (number of events for each outcome). Since all outcomes of interest were binary, the $2 * 2$ tables for each outcome were extracted. If multiple arms of the same drug at different doses were included in the same trial, the arms were combined into a single arm. This method is recommended in the Cochrane handbook [23] and was adopted in our previous meta-analysis [25]. When multiple studies of the same trial were found, the most updated publication/record was included. Data on outcomes of interest reported as SAEs on ClinicalTrials.gov were retrieved; data from the original trial publication or secondary analyses of the same trial were retrieved if no data could be extracted from ClinicalTrials.gov.

To assess methodological quality, the Cochrane Collaboration's tool for assessing risk of bias was used [26]. Bias was assessed from seven domains: random sequence generation, allocation concealment, blinding of participants and personnel, blinding of outcome assessment, incomplete outcome data, selective reporting, and other biases. In each domain, bias was judged as high, low, or unclear. The overall risk of bias was judged as high if any domain was judged as high, as low if all domains were judged as 
low, or as unclear otherwise. The certainty of evidence was assessed using the Grading of Recommendations Assessment, Development, and Evaluation (GRADE) [27].

Study selection, data extraction, and quality assessment were conducted by two independent authors (HLL, and BMYC). Any disagreement was resolved by discussion until consensus was reached, or by consulting a third author (KHY).

\section{Data synthesis and analysis}

The placebo arm was defined as the control in all analyses. Intention-to-treat analysis was employed. Relative risks (RR) and their 95\% confidence intervals (CI) were pooled using a random-effects model with inverse variance weighting. $\mathrm{RR}<1$ would favor SGLT2i over placebo. Subgroup analysis was prespecified according to the baseline condition (HF vs DM vs CKD), presence of ASCVD at baseline, the SGLT2i agent used, and follow-up duration ( $\leq$ vs $>$ median follow-up duration of all trials). Additional sensitivity analyses were performed by excluding studies with a high/unclear overall risk of bias, by excluding studies with a high/unclear bias in 'Incomplete outcome data, and by using odds ratio $(\mathrm{OR})$ as the effect measure. To minimize the unbalanced representativeness of DM-only trials, a sensitivity analysis stratifying trials into DM versus other baseline conditions was performed. Statistical heterogeneity across studies was assessed by the Cochrane's Q test and the $\mathrm{I}^{2}$ statistic. If substantial heterogeneity, as suggested by a p-value $<0.10$ or $\mathrm{I}^{2}>50 \%$, was identified, meta-regression would be used to investigate potential sources of heterogeneity. Funnel plots were used for assessment of publication bias, and Egger's test for asymmetry in funnel plot would only be performed if 10 or more studies were included [23]. Asymmetries in the funnel plots determined by visual assessment or a p-value for Egger's test $<0.10$ would suggest potential publication bias, and a trim-and-fill method was employed to adjust for potential bias. The statistical significance level was defined at 0.05 unless otherwise specified. Data analyses were performed using the "meta" package in $\mathrm{R}$ (version 3.6.3).

\section{Results}

Among the 4,532 citations identified by literature search, 22 trials [28-49] with altogether 52,115 patients $(29,211$ on SGLT2i and 22,904 on placebo) were included. The PRISMA flow diagram is shown in Fig. 1. The mean age was 63.2 years and $64.8 \%$ were male (Table 1 ). The median follow-up duration was 1.0 years (range 0.1 to

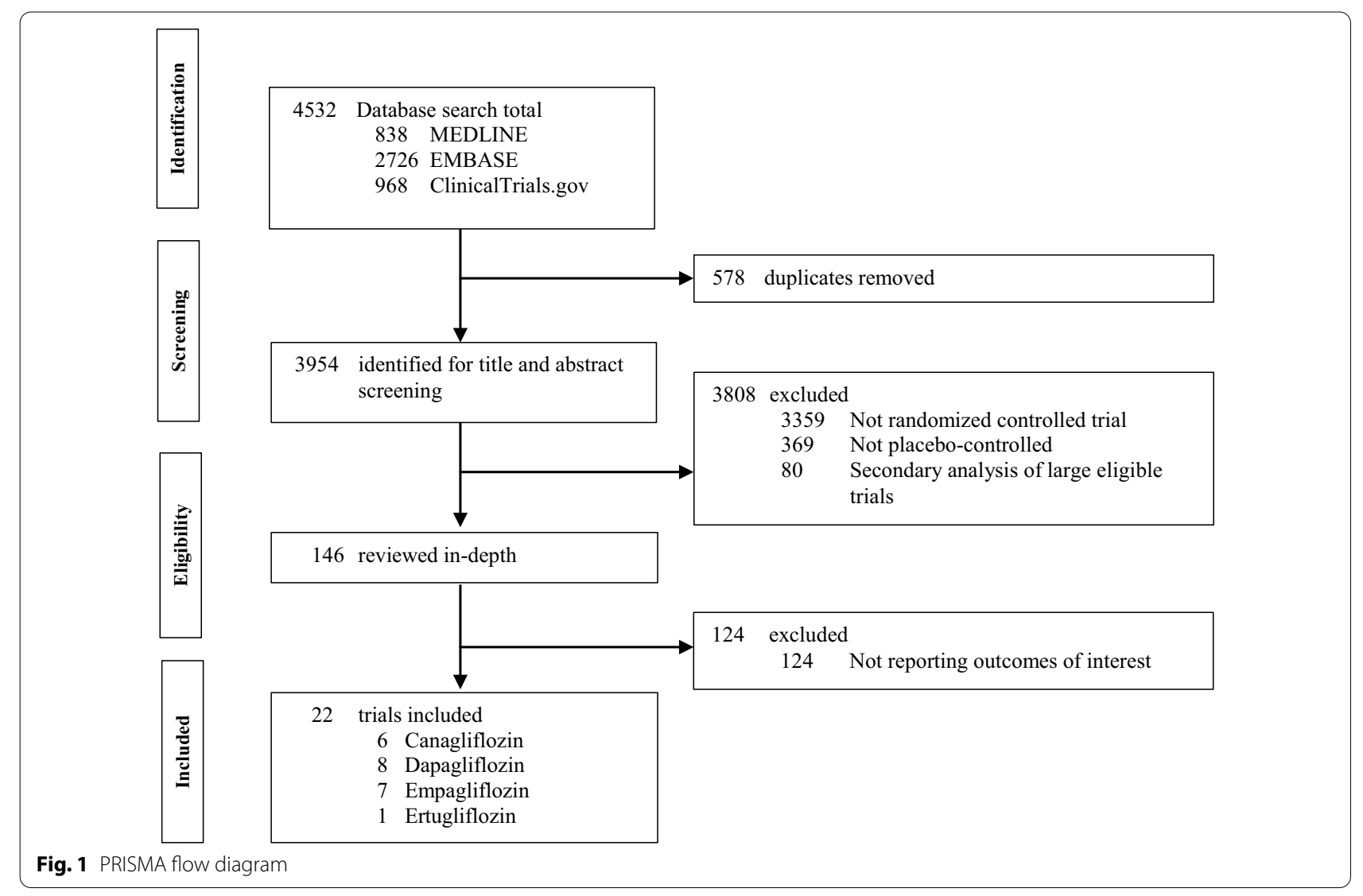




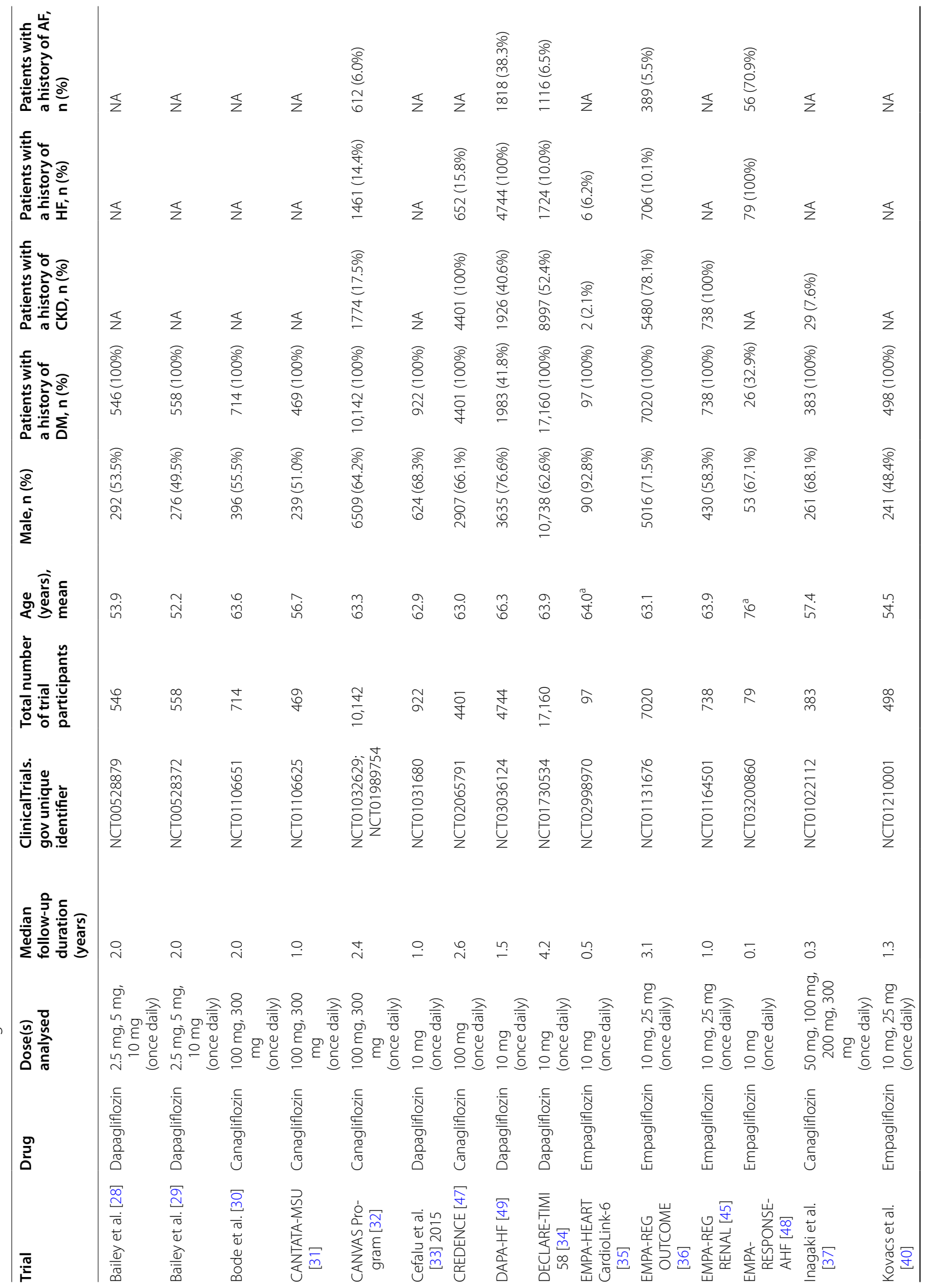




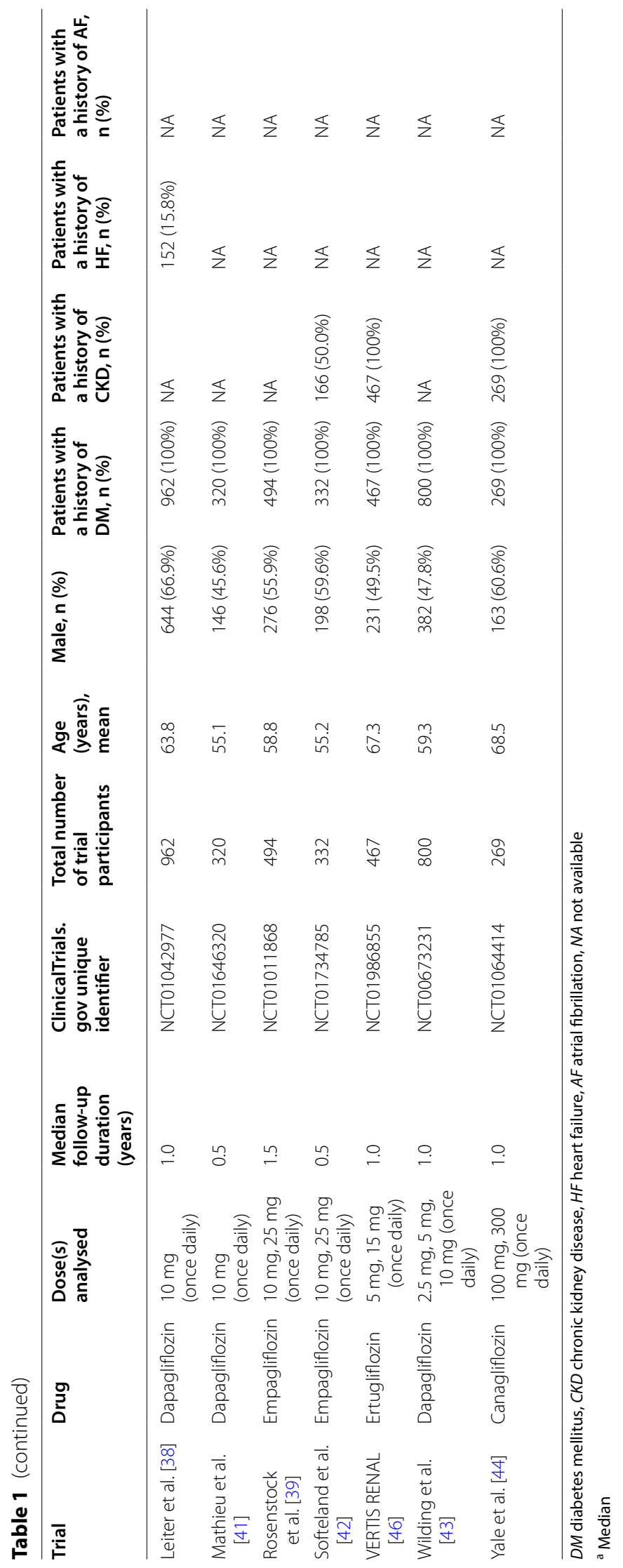


4.2). Seventeen trials enrolled patients with DM [28-44], three trials enrolled patients with DM and CKD [45-47], and two trials enrolled patients with HF [48, 49]. Overall, the percentages of the included participants with DM, CKD, and HF were $94.6 \%, 53.0 \%$ and $21.4 \%$, respectively. The mean percentage of patients with a history of AF was $10.2 \%$ (range $5.5 \%$ to $70.9 \%$ ). Eleven trials had a low risk of bias [32, 34-36, 39, 44-49], six trials had a high risk of bias $[28,29,31,33,37,43]$, and five trials had an unclear risk of bias [30, 38, 40-42] (Additional file 1: Table S3).

In total, 590 and 17 events of $\mathrm{AF}$ and embolic stroke were reported as SAEs, respectively. The RRs for AF ranged from 0.05 to 3.00 , while RRs for embolic stroke ranged from 0.17 to 2.50 . Overall, SGLT2i were associated with a $18 \%$ and $68 \%$ risk reduction in AF (RR $0.82,95 \%$ CI $0.70-0.96$ ) and embolic stroke (RR 0.32, 95\% CI 0.12-0.85) compared to placebo (Fig. 2a, b). There was no significant heterogeneity across trials $(\mathrm{p}=0.94$ and $\mathrm{p}=0.99$ for $\mathrm{AF}$ and embolic stroke, respectively). In subgroup analysis according to the baseline condition (DM vs CKD vs HF), no significant between-subgroup heterogeneity was identified $(\mathrm{p}=0.63$ and $\mathrm{p}=0.99$ for $\mathrm{AF}$ and embolic stroke, respectively) (Table 2). Similarly, there were no significant heterogeneity in subgroup analysis according to the presence of ASCVD ( $p=0.16$ and $\mathrm{p}=0.53$ for $\mathrm{AF}$ and embolic stroke, respectively). In subgroup analysis according to the SGLT2i agent used, no significant between-subgroup heterogeneity a Atrial fibrillation (AF)

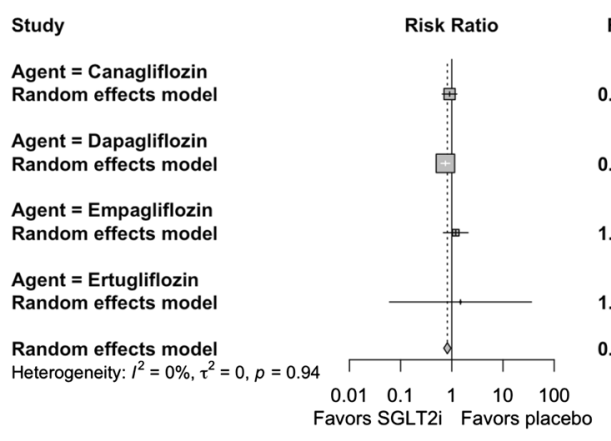

b Embolic stroke

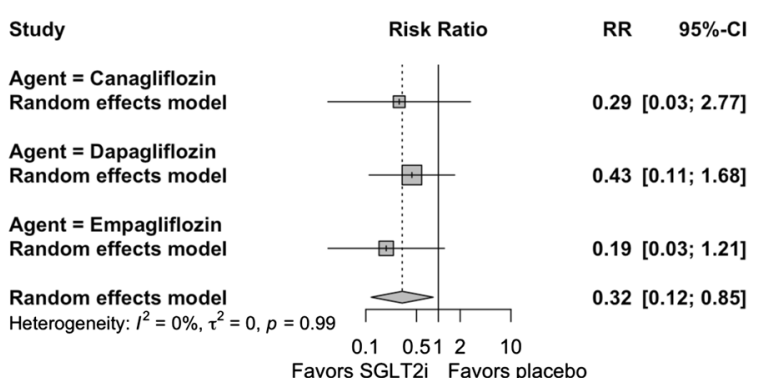

c Atrial flutter (AFL)

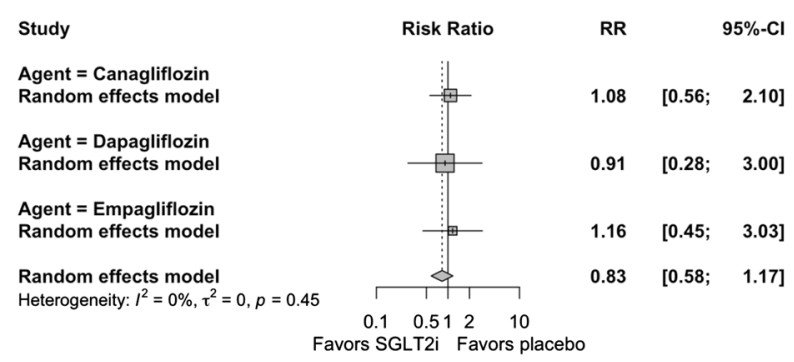

Fig. 2 Forest plots of primary analysis

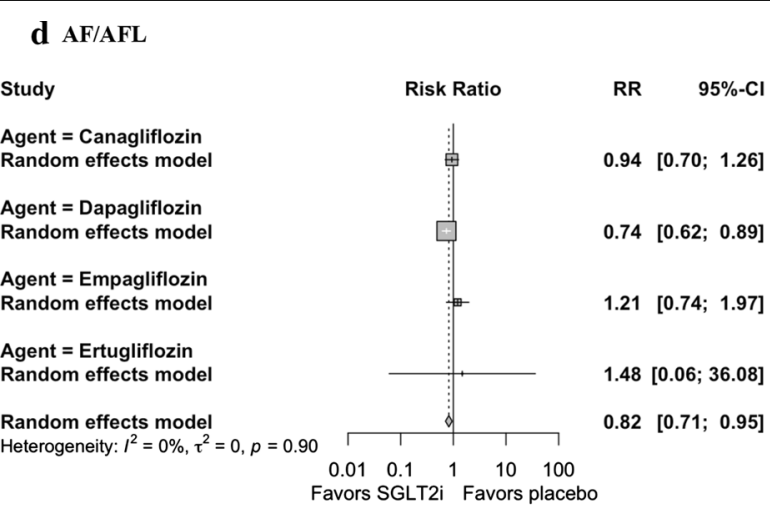

e Ventricular tachycardia (VT)

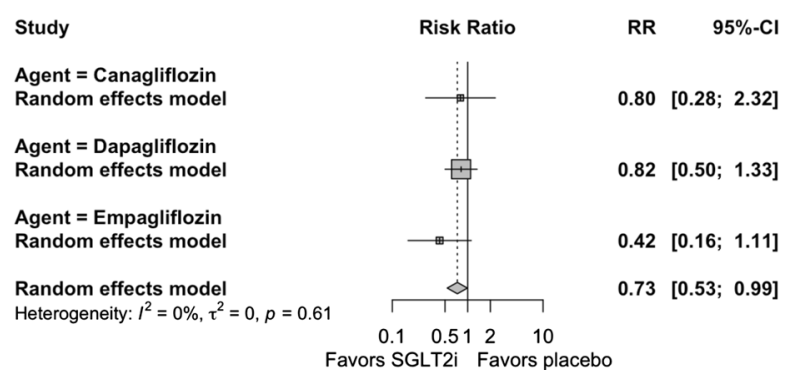

f Cardiac arrest

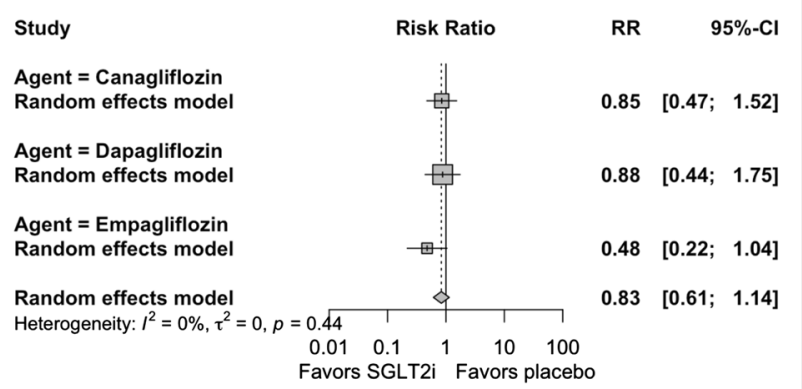


Table 2 Results of subgroup analysis

\begin{tabular}{|c|c|c|c|c|c|c|}
\hline Outcome & Subgroup & & $\begin{array}{l}\text { Number of } \\
\text { trials }\end{array}$ & $\begin{array}{l}\text { Number of } \\
\text { participants }\end{array}$ & $\mathrm{RR}(95 \% \mathrm{Cl})$ & $\overline{P_{\text {hetero }}}$ \\
\hline \multirow[t]{12}{*}{ AF } & Overall & & 21 & 51,193 & $0.82(0.70-0.96)$ & \\
\hline & Baseline condition & DM & 16 & 44,896 & $0.84(0.71-1.00)$ & 0.63 \\
\hline & & CKD & 3 & 1474 & $0.93(0.18-4.68)$ & \\
\hline & & $\mathrm{HF}$ & 2 & 4823 & $0.65(0.40-1.07)$ & \\
\hline & ASCVD & No ASCVD & 18 & 43,114 & $0.79(0.67-0.94)$ & 0.16 \\
\hline & & ASCVD present & 3 & 8079 & $1.23(0.69-2.19)$ & \\
\hline & SGLT2i agent & Canagliflozin & 6 & 16,378 & $0.90(0.65-1.26)$ & 0.39 \\
\hline & & Dapagliflozin & 7 & 25,090 & $0.75(0.61-0.91)$ & \\
\hline & & Empagliflozin & 7 & 9258 & $1.19(0.68-2.08)$ & \\
\hline & & Ertugliflozin & 1 & 467 & $1.48(0.06-36.08)$ & \\
\hline & Follow-up duration & $\leq 1$ year & 11 & 4916 & $0.82(0.69-0.97)$ & 0.90 \\
\hline & & $>1$ year & 10 & 42,677 & $0.82(0.70-0.96)$ & \\
\hline \multirow[t]{11}{*}{ Embolic stroke } & Overall & & 6 & 44,205 & $0.32(0.12-0.85)$ & \\
\hline & Baseline condition & $\mathrm{DM}$ & 4 & 38,723 & $0.32(0.10-1.03)$ & 0.99 \\
\hline & & CKD & 1 & 4401 & $0.25(0.01-6.21)$ & \\
\hline & & $\mathrm{HF}$ & 1 & 4744 & $0.25(0.03-3.20)$ & \\
\hline & ASCVD & No ASCVD & 5 & 37,185 & $0.37(0.12-1.10)$ & 0.53 \\
\hline & & ASCVD present & 1 & 7020 & $0.17(0.02-1.59)$ & \\
\hline & SGLT2i agent & Canagliflozin & 2 & 14,543 & $0.29(0.03-2.77)$ & 0.78 \\
\hline & & Dapagliflozin & 3 & 21,904 & $0.43(0.11-1.68)$ & \\
\hline & & Empagliflozin & 4 & 7758 & $1.19(0.03-1.21)$ & \\
\hline & Follow-up duration & $\leq 1$ year & 1 & 738 & $0.25(0.01-6.21)$ & 0.89 \\
\hline & & $>1$ year & 5 & 43,467 & $0.32(0.12-0.92)$ & \\
\hline \multirow[t]{11}{*}{ AFL } & Overall & & 9 & 45,478 & $0.83(0.58-1.17)$ & \\
\hline & Baseline condition & DM & 6 & 36,333 & $0.75(0.52-1.09)$ & 0.19 \\
\hline & & CKD & 1 & 4401 & $1.00(0.14-7.08)$ & \\
\hline & & HF & 1 & 4744 & $2.66(0.71-10.03)$ & \\
\hline & ASCVD & No ASCVD & 6 & 37,439 & $0.91(0.55-1.48)$ & 0.90 \\
\hline & & ASCVD present & 3 & 8039 & $0.97(0.36-2.66)$ & \\
\hline & SGLT2i agent & Canagliflozin & 2 & 14,543 & $1.08(0.56-2.10)$ & 0.95 \\
\hline & & Dapagliflozin & 3 & 22,826 & $0.91(0.28-3.00)$ & \\
\hline & & Empagliflozin & 4 & 8109 & $1.14(0.45-3.03)$ & \\
\hline & Follow-up duration & $\leq 1$ year & 2 & 1019 & $0.33(0.01-8.20)$ & 0.56 \\
\hline & & $>1$ year & 7 & 44,459 & $0.87(0.59-1.29)$ & \\
\hline \multirow[t]{12}{*}{ AF/AFL } & Overall & & 22 & 49,115 & $0.82(0.71-0.95)$ & \\
\hline & Baseline condition & $\mathrm{DM}$ & 17 & 41,686 & $0.82(0.70-0.97)$ & 0.99 \\
\hline & & CKD & 3 & 5606 & $0.83(0.46-1.51)$ & \\
\hline & & $\mathrm{HF}$ & 2 & 4823 & $0.79(0.51-1.24)$ & \\
\hline & ASCVD & No ASCVD & 18 & 40,114 & $0.79(0.68-0.93)$ & 0.16 \\
\hline & & ASCVD present & 4 & 9001 & $1.16(0.70-1.91)$ & \\
\hline & SGLT2i agent & Canagliflozin & 6 & 16,378 & $0.94(0.70-1.26)$ & 0.20 \\
\hline & & Dapagliflozin & 8 & 26,012 & $0.74(0.62-0.89)$ & \\
\hline & & Empagliflozin & 7 & 9258 & $1.21(0.74-1.97)$ & \\
\hline & & Ertugliflozin & 1 & 467 & $1.48(0.06-36.08)$ & \\
\hline & Follow-up duration & $\leq 1$ year & 12 & 5838 & $0.73(0.31-1.71)$ & 0.78 \\
\hline & & $>1$ year & 10 & 42,677 & $0.82(0.71-0.96)$ & \\
\hline
\end{tabular}


Table 2 (continued)

\begin{tabular}{|c|c|c|c|c|c|c|}
\hline Outcome & Subgroup & & $\begin{array}{l}\text { Number of } \\
\text { trials }\end{array}$ & $\begin{array}{l}\text { Number of } \\
\text { participants }\end{array}$ & RR $(95 \% \mathrm{Cl})$ & $\overline{P_{\text {hetero }}}$ \\
\hline \multirow[t]{11}{*}{$\overline{V T}$} & Overall & & 7 & 43,963 & $0.73(0.53-0.99)$ & \\
\hline & \multirow[t]{3}{*}{ Baseline condition } & DM & 4 & 34,739 & $0.90(0.56-1.42)$ & \multirow[t]{3}{*}{0.50} \\
\hline & & CKD & 1 & 2501 & $0.50(0.05-5.50)$ & \\
\hline & & $\mathrm{HF}$ & 2 & 4823 & $0.62(0.41-0.95)$ & \\
\hline & \multirow[t]{2}{*}{ ASCVD } & No ASCVD & 6 & 36,846 & $0.77(0.56-1.06)$ & \multirow[t]{2}{*}{0.30} \\
\hline & & ASCVD present & 2 & 7117 & $0.44(0.16-1.20)$ & \\
\hline & \multirow[t]{3}{*}{ SGLT2i agent } & Canagliflozin & 2 & 14,543 & $0.80(0.28-2.32)$ & \multirow[t]{3}{*}{0.48} \\
\hline & & Dapagliflozin & 3 & 22,224 & $0.82(0.50-1.33)$ & \\
\hline & & Empagliflozin & 3 & 7196 & $0.42(0.16-1.11)$ & \\
\hline & \multirow[t]{2}{*}{ Follow-up duration } & $\leq 1$ year & 3 & 496 & $0.98(0.10-9.29)$ & \multirow[t]{2}{*}{0.79} \\
\hline & & $>1$ year & 5 & 43,647 & $0.72(0.53-0.99)$ & \\
\hline \multirow[t]{11}{*}{ Cardiac arrest } & Overall & & 7 & 44,751 & $0.83(0.61-1.14)$ & \\
\hline & \multirow[t]{3}{*}{ Baseline condition } & DM & 4 & 34,868 & $0.74(0.42-1.30)$ & \multirow[t]{3}{*}{0.93} \\
\hline & & CKD & 2 & 5139 & $0.72(0.25-2.08)$ & \\
\hline & & HF & 1 & 4744 & $0.90(0.37-2.21)$ & \\
\hline & \multirow[t]{2}{*}{ ASCVD } & No ASCVD & 6 & 37,731 & $0.92(0.66-1.30)$ & \multirow[t]{2}{*}{0.12} \\
\hline & & ASCVD present & 1 & 7020 & $0.46(0.20-1.03)$ & \\
\hline & \multirow[t]{3}{*}{ SGLT2i agent } & Canagliflozin & 2 & 14,543 & $0.85(0.46-1.52)$ & \multirow[t]{3}{*}{0.44} \\
\hline & & Dapagliflozin & 3 & 22,450 & $0.88(0.44-1.75)$ & \\
\hline & & Empagliflozin & 2 & 7758 & $0.48(0.22-1.04)$ & \\
\hline & \multirow[t]{2}{*}{ Follow-up duration } & $\leq 1$ year & 1 & 738 & $0.76(0.05-12.13)$ & \multirow[t]{2}{*}{0.97} \\
\hline & & $>1$ year & 6 & 44,013 & $0.81(0.56-1.16)$ & \\
\hline
\end{tabular}

$A F$ atrial fibrillation, $A F L$ atrial flutter, $V T$ ventricular tachycardia, $R R$ risk ratio, 95\% Cl 95\% confidence interval, $P_{\text {hetero }}$ P-value for between-subgroup heterogeneity, DM diabetes mellitus, CKD chronic kidney disease, HF heart failure, ASCVD atherosclerotic cardiovascular disease

was identified $(\mathrm{p}=0.39$ and $\mathrm{p}=0.78$ for $\mathrm{AF}$ and embolic stroke, respectively). There were no significant heterogeneity in subgroup analysis according to follow-up duration $(\mathrm{p}=0.90$ and $\mathrm{p}=0.89$ for $\mathrm{AF}$ and embolic stroke, respectively).

A total of 135 events of AFL were reported as SAEs. The RRs for AFL ranged from 0.33 to 2.66. Overall, SGLT2i did not significantly influence the risk of AFL (RR 0.83, 95\% CI 0.68-1.17) compared to placebo (Fig. 2c). There was no significant heterogeneity across trials $(p=0.45)$. In subgroup analysis according to baseline condition, according to the presence of ASCVD, according to the SGLT2i agent used, and according to follow-up duration, no significant between-subgroup heterogeneity was identified $(\mathrm{p}=0.19, \mathrm{p}=0.90, \mathrm{p}=0.95$, and $\mathrm{p}=0.56$, respectively) (Table 2).

When AF and AFL are combined as a composite endpoint, SGLT2i are associated with an $18 \%$ risk reduction in AF/AFL (RR 0.82, 95\% CI 0.71-0.95) (Fig. 2d). There was no significant heterogeneity across trials $(\mathrm{p}=0.90)$. Subgroup analysis stratifying studies according to baseline condition, presence of ASCVD, SGLT2i agent, and follow-up duration did not identify a significant between-subgroup heterogeneity $(p=0.99, p=0.16$, $\mathrm{p}=0.20$, and $\mathrm{p}=0.78$, respectively) (Table 2 ).

A total of 163 events of VT were reported as SAEs. The RRs for VT ranged from 0.33 to 3.00. Overall, SGLT2i were associated with a $27 \%$ risk reduction in VT compared to placebo (RR 0.73, 95\% CI 0.53-0.99) (Fig. 2e). There was no significant heterogeneity across trials $(\mathrm{p}=0.61)$. Subgroup analysis stratifying studies according to baseline condition, presence of ASCVD, SGLT2i agent, and follow-up duration did not identify a significant between-subgroup heterogeneity $(\mathrm{p}=0.50, \mathrm{p}=0.30$, $\mathrm{p}=0.48$, and $\mathrm{p}=0.79$, respectively) (Table 2 ).

A total of 157 cardiac arrest events were reported as SAEs. The RRs for cardiac arrest ranged from 0.07 to 1.06. Overall, SGLT2i did not significantly influence the risk of cardiac arrest (RR 0.83 , 95\% CI 0.61-1.14) (Fig. 2f). There was no significant heterogeneity across trials $(p=0.44)$. Subgroup analysis according to baseline condition, presence of ASCVD, SGLT2i agent, and 
follow-up duration did not identify a significant betweensubgroup heterogeneity $(\mathrm{p}=0.93, \mathrm{p}=0.12, \mathrm{p}=0.44$, and $\mathrm{p}=0.97$, respectively) (Table 2 ).

Sensitivity analyses excluding studies with a high/ unclear overall risk of bias, excluding studies with a high/ unclear risk of bias in Incomplete outcome data, and using OR as an effect measure yielded largely consistent results (Additional file 1: Table S4(A)). In the sensitivity analysis stratifying trials according to DM versus other baseline conditions (CKD or HF), there were no significant subgroup differences (Additional file 1: Table S4(B)). Symmetry was observed in the funnel plots for AF, embolic stroke, AF/AFL, VT, and cardiac arrest, but not for AFL (Additional file 1: Fig. S1). Egger's test for AF and AF/AFL did not reveal significant asymmetry, whereas Egger's test was not performed for embolic stroke, AFL, $\mathrm{VT}$, and cardiac arrest since the number of studies was below 10. Trim-and-fill method generated an overall RR of 0.77 (95\% CI 0.52-1.14) for AFL. The GRADE assessment for each outcome was shown in Additional file 1: Table S5. The certainty of evidence for AF, embolic stroke, AF/AFL, and VT were graded as high, whereas the certainty for AFL and cardiac arrest were graded as moderate due to imprecision (as the $95 \%$ of the relative risk was sufficiently wide that the estimate could include appreciable benefit/harm of the use of SGLT2i, with 0.75 and 1.25 taken as thresholds).

\section{Discussion}

In this systematic review and meta-analysis of 22 trials with 52,115 patients with DM, CKD, or HF susceptible to developing arrhythmias, we found that SGLT2i treatment might be associated with a lower risk of $\mathrm{AF}$, embolic stroke, AF/AFL, and VT, compared to placebo. The associations appeared to be consistent across all baseline conditions (HF vs DM vs CKD; ASCVD vs no ASCVD), all SGLT2i subgroups, and across short vs long followup duration. Although no significant associations were observed for AFL and cardiac arrest, the point estimates appeared to be consistent with that of AF. These findings are consistent with recent reports suggesting that SGLT2i reduced the risk of arrhythmias $[17,20]$.

To the authors' knowledge, this is the largest and most comprehensive systematic review and meta-analysis that addresses the association between SGLT2i and arrhythmia outcomes.

A previous meta-analysis did not find a significant association between SGLT2i treatment and AF (OR 0.61, 95\% CI 0.31-1.19) [18]. As the number of participants $(52,115$ vs 10,512$)$ and events (590 vs 30$)$ are much larger in our meta-analysis, the association we identified, which suggests a significant risk reduction in AF with SGLT2i treatment, is more likely to be robust. In the EMPA-REG
OUTCOME trial, although the incidence of new-onset AF appeared to be higher in the SGLT2i group (2.3\%) than in the placebo group (1.6\%), the difference did not reach statistical significance [19]. Furthermore, no significant difference between SGLT2i subgroups was identified in our meta-analysis. Previous meta-analyses have identified consistent risk reductions in adverse cardiovascular/renal events across different SGLT2i agents, and empagliflozin is likely to exhibit similar cardio- and renoprotective properties $[14,50]$. Nevertheless, a recent real-world cohort study of patients with DM found that empagliflozin resulted in poorer outcome in reduction of HF compared to dapagliflozin [51]. Therefore, larger studies evaluating the effects of empagliflozin on AF are required to confirm the association.

A previous meta-analysis found that SGLT2i did not significantly influence the risk of stroke [52]. However, embolic stroke was not specifically studied as an outcome. No previous studies have evaluated the association between SGLT2i and embolic stroke, and the current meta-analysis is the first to report the protective effect of SGLT2i on embolic stroke. Such association might be attributed to the risk reduction in AF. Nevertheless, owing to the low number of events reported in the included trials, larger studies evaluating the association between SGLT2i and embolic stroke are needed to confirm our findings.

Although our meta-analysis failed to identify a significant risk reduction in AFL, a statistically significant risk reduction was identified when AF and AFL were evaluated as a composite outcome. A similar risk reduction in AF/AFL events (RR 0.81, 95\% CI 0.67-0.98) was identified in the secondary analysis of the DECLARE-TIMI 58 trial [17]. The low number of AFL events observed in the included trials in our meta-analysis might have contributed to a wide confidence interval, hence a marginally significant association. Larger RCTs powered to detect differences in AFL are required to confirm these findings. Nevertheless, AF and AFL have similar clinical significance and consequences, [53] and the conjoint analysis of AF/AFL, which shows a significant risk reduction, provides more robust results while obviating possible publication bias for AFL.

A previous meta-analysis by $\mathrm{Li}$ et al. identified a $24 \%$ risk reduction in AF/AFL with SGLT2i treatment, [54] as compared to $18 \%$ and $17 \%$ risk reduction in $\mathrm{AF}$ and $\mathrm{AFL}$, respectively, in our meta-analysis. The inconsistency could be explained by the significant methodological differences. In addition to trials of DM patients, our meta-analysis also included trials of $\mathrm{HF}$ and CKD patients, resulting in a significantly higher number of trials (22 vs 16$)$ and patients $(52,115$ vs 38,335$)$ included. Therefore, our observations are likely to be more robust 
and accurate. More importantly, as the use of SGLT2i has greatly expanded from selected DM patients only to patients with DM, CKD, and cardiovascular diseases, [55] our findings are applicable to patients with a much broader spectrum of comorbidities. Furthermore, the meta-analysis by Li et al. only evaluated AF/AFL as an arrhythmia outcome, as compared to four additional outcomes (AF, embolic stroke, AFL, VT, and cardiac arrest) evaluated in our meta-analysis, highlighting the comprehensiveness of the present study. Our meta-analysis provides a more holistic evaluation of how SGLT2i reduced the risk of arrhythmias.

The association between SGLT2i, VT, and cardiac arrest has been less well studied. To our knowledge, this meta-analysis is the first study to address this research question. SGLT2i treatment was associated with a $28 \%$ risk reduction for VT. In the recent EMBODY trial, [56] improvements in indicators of cardiac sympathetic/ parasympathetic nerve activity, which are related to the risk of ventricular tachyarrhythmias, were greater in the empagliflozin group compared to the placebo group. Only the empagliflozin group achieved a significant intragroup improvement. Taking these findings together, it is likely that SGLT2i may exert a protecting effect against VT. Meanwhile, six out of seven studies reported an RR of $<1.0$ for cardiac arrest, and there was a $17 \%$ risk reduction in developing cardiac arrest. The marginally significant association could be attributed to a low number of events, and larger prospective studies are warranted to confirm the association.

It is being increasingly recognized that HF, DM, and CKD are associated with AF and cardiac arrhythmias $[1-4,9]$. The presence of AF is associated with a higher risk of adverse cardiovascular events, cardiovascular mortality, and all-cause mortality in individuals with HF, CKD, and DM [2, 4, 7-9]. Similar associations were observed for AFL and VT: HF and DM may predispose to the development of AFL and VT, which are in turn associated with higher mortality [10-12]. Therefore, it is of critical importance to reduce the risk of arrhythmias in patients with HF, DM, and CKD.

The pathophysiological pathways linking DM, CKD, and HF with the development of AF and arrhythmias are complex and multifactorial $[2,3,6]$. The presence of DM has been implicated to explain the coexistence of CKD, $\mathrm{HF}$, and $\mathrm{AF}[3,4]$. Compared to the general population, individuals with DM and stage 5 CKD have a threefold increased risk to develop AF, [5] in which comorbid DM increases the risk of bleeding in patients with AF [57]. DM has also been found to increase the risk of suboptimal response to cardiac resynchronization therapy with defibrillator in patients with HF [58]. Furthermore, multiple signaling pathways contribute to remodeling and arrhythmogenic properties in HF, increasing the risk of developing ventricular tachyarrhythmias $[9,59]$. There are also several possible mechanisms through which SGLT2i reduce the risk of arrhythmias. By promoting osmotic diuresis and natriuresis, SGLT2i alleviate cardiac workload and improve left ventricular function [60]. SGLT2i may also reduce arrhythmia by modulating neurohormonal pathways, which, in DM, CKD, and HF, are activated and play important roles in the deterioration of these conditions [61, 62]. By optimizing hemodynamic status, SGLT2i reduce fluid overload, which is associated with cardiac structural abnormalities, hence predisposition to arrhythmia, in DM and CKD [63]. SGLT2i are also effective in blood pressure and glycemic control, both of which are implicated in cardiac arrhythmogenesis [64]. Furthermore, by inhibiting the myocardial sodiumhydrogen exchanger $\left(\mathrm{Na}^{+} / \mathrm{H}^{+}\right.$exchanger $)$, which is upregulated in HF, SGLT2i lead to improvement in mitochondrial dysfunction and reduction in oxidative stress, thus reducing the risk of arrhythmias $[65,66]$. Apart from improving mitochondrial function, [67] SGLT2i have also been found to alleviate atrial remodeling, an important process implicated in atrial arrhythmogenesis [68]. SGLT2i may also reduce electrical instability by ensuring a sufficient energy supply [69-71]. Other possible mechanisms are outlined in Additional file 1: Fig. S2.

There are several limitations in this meta-analysis. First, arrhythmia outcomes were not the pre-specified outcomes of the included trials, and there might be ascertainment bias. The outcomes were not adjudicated and might lead to inaccuracies and incompleteness of data. Nevertheless, in sensitivity analyses where studies with high/unclear overall risk of bias and studies with high/unclear risk of bias in 'Incomplete outcome data' were excluded, largely consistent associations were observed. Nevertheless, the approach of using adverse events as outcomes has been used in previous studies $[21,54]$. Nonetheless, further randomized trials with well-defined and adjudicated arrhythmia outcomes are required to confirm the associations reported in the current study. Second, the included trials were underpowered to detect differences in arrhythmia outcomes. Future trials designed with arrhythmias as the primary outcomes are warranted. Third, outcomes on arrhythmia-related mortality were not included. These outcomes are clinically more important but were not reported in the identified trials. AF confers higher mortality in patients with DM, CKD, and HF, and further studies examining the effects of SGLT2i on arrhythmiarelated death are urgently needed. Fourth, as patientlevel data were not available and not all trials reported the baseline prevalence of $\mathrm{DM} / \mathrm{CKD} / \mathrm{HF}$, trials could not be grouped according to a combination of disease 
processes, for instance, $\mathrm{DM}+\mathrm{CKD}+\mathrm{HF}$. Therefore, further studies are required to evaluate whether SGLT2 inhibitors could reduce incidences of arrhythmia in patients with multiple comorbidities. Fifth, as no data on the number of events specific to baseline comorbidity, for instance, the number of AF events in those with pre-existing HF vs those without, were available from the trial, the results of subgroup analyses should be interpreted with caution, and further studies are warranted to confirm that the associations between SGLT2i and arrhythmias remain significant regardless of the presence of baseline comorbidity. Sixth, patient-level data on pre-existing AF and the use of anti-arrhythmic medications were not available from most trials.

\section{Conclusions}

This meta-analysis found that SGLT2i reduced the risk of AF and VT. Our study provides further robust evidence for recommending the use of SGLT2i in patients with DM, CKD, and HF to reduce related cardiac complications and comorbidities. However, the mechanisms by which SGLT2i protects against arrhythmias are complex and further research is warranted.

\section{Abbreviations}

AF: Atrial fibrillation; AFL: Atrial flutter; ASCVD: Atherosclerotic cardiovascular disease; CKD: Chronic kidney disease; DM: Diabetes mellitus; HF: Heart failure; OR: Odds ratio; RR: Relative risk; SAEs: Serious adverse events; SGLT2i: Sodiumglucose cotransporter 2 inhibitors; VT: Ventricular tachycardia.

\section{Supplementary Information}

The online version contains supplementary material available at https://doi. org/10.1186/s12933-021-01293-8.

Additional file 1: Figure S1. Funnel plots and results of Egger's test for asymmetry. Figure S2. Proposed mechanisms through which SGLT2 inhibitors reduce risk of arrhythmias. Table S1. PRISMA checklist. Table S2. Search strategy. Table S3. Risk of bias assessment. Table S4. Results of sensitivity analyses. Table S5. GRADE assessment.

\section{Acknowledgements}

Supported by the Shenzhen Key Medical Discipline (No.SZXK2020081), and The Sanming Project of HKU-SZH Cardiology (SZSM201911020).

\section{Authors' contributions}

Study concept and design: HLL, GYHL, QF, FY, BMYC, KHY. Acquisition, analysis, or interpretation of data: All authors. Drafting of manuscript: HLL. Critical revision of the manuscript for important intellectual content: QF, FY, GYHL, BMYC, KHY. Statistical analysis: HLL. Supervision: BMYC, KHY. All authors read and approved the final manuscript.

\section{Funding}

None.

\section{Availability of data and materials}

The review has not been registered; The dataset(s) supporting the conclusions of this article is(are) included within the article.

\section{Declarations}

Ethics approval and consent to participate

Not applicable.

\section{Consent for publication}

Not applicable.

\section{Competing interests}

The authors declare that they have no competing interests.

\section{Author details}

${ }^{1}$ Division of Cardiology, Department of Medicine, The University of Hong Kong, Queen Mary Hospital, Room 1929B/K1931, Block K, Hong Kong, China. ${ }^{2}$ Liverpool Centre for Cardiovascular Science, University of Liverpool and Liverpool Heart \& Chest Hospital, Liverpool, UK. ${ }^{3}$ Aalborg Thrombosis Research Unit, Department of Clinical Medicine, Aalborg University, Aalborg, Denmark. ${ }^{4}$ Jockey Club School of Public Health and Primary Care, The Chinese University of Hong Kong, Hong Kong, China. ${ }^{5}$ Division of Clinical Pharmacology, Department of Medicine, The University of Hong Kong, Queen Mary Hospital, Hong Kong, China. ${ }^{6}$ Division of Cardiology, Department of Medicine, The University of Hong Kong Shenzhen Hospital, Shenzhen, China.

Received: 25 March 2021 Accepted: 27 April 2021

Published online: 07 May 2021

\section{References}

1. Lip GY, Tse HF, Lane DA. Atrial fibrillation. Lancet. 2012;379(9816):648-61.

2. Wang A, Green JB, Halperin JL, Piccini JP Sr. Atrial fibrillation and diabetes mellitus: JACC review topic of the week. J Am Coll Cardiol. 2019;74(8):1107-15.

3. Anter E, Jessup M, Callans DJ. Atrial fibrillation and heart failure: treatment considerations for a dual epidemic. Circulation. 2009;1 19(18):2516-25.

4. Kumar S, Lim E, Covic A, Verhamme P, Gale CP, Camm AJ, et al. Anticoagulation in concomitant chronic kidney disease and atrial fibrillation: JACC review topic of the week. J Am Coll Cardiol. 2019;74(17):2204-15.

5. Seyed Ahmadi S, Svensson AM, Pivodic A, Rosengren A, Lind M. Risk of atrial fibrillation in persons with type 2 diabetes and the excess risk in relation to glycaemic control and renal function: a Swedish cohort study. Cardiovasc Diabetol. 2020;19(1):9.

6. Grisanti LA. Diabetes and arrhythmias: pathophysiology. Mech Ther Outcomes Front Physiol. 2018;9:1669.

7. Wang TJ, Larson MG, Levy D, Vasan RS, Leip EP, Wolf PA, et al. Temporal relations of atrial fibrillation and congestive heart failure and their joint influence on mortality: the Framingham Heart Study. Circulation. 2003;107(23):2920-5.

8. Middlekauff HR, Stevenson WG, Stevenson LW. Prognostic significance of atrial fibrillation in advanced heart failure. A study of 390 patients. Circulation. 1991;84(1):40-8.

9. Lip GY, Heinzel FR, Gaita F, Juanatey JR, Le Heuzey JY, Potpara T, et al. European Heart Rhythm Association/Heart Failure Association joint consensus document on arrhythmias in heart failure, endorsed by the Heart Rhythm Society and the Asia Pacific Heart Rhythm Society. Europace. 2016;18(1):12-36.

10. Granada J, Uribe W, Chyou PH, Maassen K, Vierkant R, Smith PN, et al. Incidence and predictors of atrial flutter in the general population. J Am Coll Cardiol. 2000;36(7):2242-6.

11. Baldasseroni S, Opasich C, Gorini M, Lucci D, Marchionni N, Marini M, et al. Left bundle-branch block is associated with increased 1-year sudden and total mortality rate in 5517 outpatients with congestive heart failure: a report from the Italian network on congestive heart failure. Am Heart J. 2002;143(3):398-405.

12. Tran HV, Gore JM, Darling CE, Ash AS, Kiefe Cl, Goldberg RJ. Hyperglycemia and risk of ventricular tachycardia among patients hospitalized with acute myocardial infarction. Cardiovasc Diabetol. 2018;17(1):136.

13. Zelniker TA, Braunwald E. Cardiac and renal effects of sodium-glucose co-transporter 2 inhibitors in diabetes: JACC state-of-the-art review. J Am Coll Cardiol. 2018;72(15):1845-55. 
14. Arnott C, Li Q, Kang A, Neuen BL, Bompoint S, Lam CSP, et al. Sodiumglucose cotransporter 2 inhibition for the prevention of cardiovascular events in patients with type 2 diabetes mellitus: a systematic review and meta-analysis. J Am Heart Assoc. 2020;9(3):e014908.

15. Fei Y, Tsoi MF, Cheung BMY. Cardiovascular outcomes in trials of new antidiabetic drug classes: a network meta-analysis. Cardiovasc Diabetol. 2019;18(1):112.

16. Baker WL, Smyth LR, Riche DM, Bourret EM, Chamberlin KW, White WB. Effects of sodium-glucose co-transporter 2 inhibitors on blood pressure: a systematic review and meta-analysis. J Am Soc Hypertens. 2014;8(4):262-75.e9.

17. Zelniker TA, Bonaca MP, Furtado RHM, Mosenzon O, Kuder JF, Murphy SA, et al. Effect of dapagliflozin on atrial fibrillation in patients with type 2 diabetes mellitus: insights from the DECLARE-TIMI 58 trial. Circulation. 2020;141(15):1227-34.

18. Usman MS, Siddiqi TJ, Memon MM, Khan MS, Rawasia WF, Talha Ayub M, et al. Sodium-glucose co-transporter 2 inhibitors and cardiovascular outcomes: a systematic review and meta-analysis. Eur J Prev Cardiol. 2018;25(5):495-502.

19. Böhm M, Slawik J, Brueckmann M, Mattheus M, George JT, Ofstad AP, et al. Efficacy of empagliflozin on heart failure and renal outcomes in patients with atrial fibrillation: data from the EMPA-REG OUTCOME trial. Eur J Heart Fail. 2020;22(1):126-35.

20. Chen HY, Huang JY, Siao WZ, Jong GP. The association between SGLT2 inhibitors and new-onset arrhythmias: a nationwide population-based longitudinal cohort study. Cardiovasc Diabetol. 2020;19(1):73.

21. Bonora BM, Raschi E, Avogaro A, Fadini GP. SGLT-2 inhibitors and atrial fibrillation in the Food and Drug Administration adverse event reporting system. Cardiovasc Diabetol. 2021;20(1):39.

22. Persson F, Nyström T, Jørgensen ME, Carstensen B, Gulseth HL, Thuresson $M$, et al. Dapagliflozin is associated with lower risk of cardiovascular events and all-cause mortality in people with type 2 diabetes (CVDREAL Nordic) when compared with dipeptidyl peptidase-4 inhibitor therapy: a multinational observational study. Diabetes Obes Metab. 2018;20(2):344-51.

23. Higgins JPT, Green S. Cochrane Handbook for Systematic Reviews of Interventions. 2011

24. Page MJ, McKenzie JE, Bossuyt PM, Boutron I, Hoffmann TC, Mulrow CD, et al. The PRISMA 2020 statement: an updated guideline for reporting systematic reviews. BMJ. 2021;372:n71.

25. Li HL, Feng Q, Tsoi MF, Fei Y, Cheung BMY. Risk of infections in patients treated with ticagrelor versus clopidogrel: a systematic review and metaanalysis. Eur Heart J Cardiovasc Pharmacother. 2020. https://doi.org/10. 1093/ehjcvp/pvaa065.

26. Higgins JP, Altman DG, Gøtzsche PC, Jüni P, Moher D, Oxman AD, et al. The Cochrane Collaboration's tool for assessing risk of bias in randomised trials. BMJ. 2011;343:d5928.

27. Guyatt GH, Oxman AD, Vist GE, Kunz R, Falck-Ytter Y, Alonso-Coello P, et al. GRADE: an emerging consensus on rating quality of evidence and strength of recommendations. BMJ. 2008;336(7650):924-6.

28. Bailey CJ, Gross JL, Hennicken D, labal N, Mansfield TA, List JF. Dapagliflozin add-on to metformin in type 2 diabetes inadequately controlled with metformin: a randomized, double-blind, placebo-controlled 102-week trial. BMC Med. 2013;11:43.

29. Bailey CJ, Morales Villegas EC, Woo V, Tang W, Ptaszynska A, List JF. Efficacy and safety of dapagliflozin monotherapy in people with Type 2 diabetes: a randomized double-blind placebo-controlled 102-week trial. Diabet Med. 2015;32(4):531-41.

30. Bode B, Stenlöf K, Harris S, Sullivan D, Fung A, Usiskin K, et al. Longterm efficacy and safety of canagliflozin over 104 weeks in patients aged 55-80 years with type 2 diabetes. Diabetes Obes Metab. 2015;17(3):294-303

31. Wilding JP, Charpentier G, Hollander P, González-Gálvez G, Mathieu C, Vercruysse $F$, et al. Efficacy and safety of canagliflozin in patients with type 2 diabetes mellitus inadequately controlled with metformin and sulphonylurea: a randomised trial. Int J Clin Pract. 2013;67(12):1267-82.

32. Neal B, Perkovic V, Mahaffey KW, de Zeeuw D, Fulcher G, Erondu N, et al. Canagliflozin and Cardiovascular and Renal Events in Type 2 Diabetes. N Engl J Med. 2017;377(7):644-57.

33. Cefalu WT, Leiter LA, de Bruin TW, Gause-Nilsson I, Sugg J, Parikh SJ. Dapagliflozin's effects on glycemia and cardiovascular risk factors in high-risk patients with type 2 diabetes: a 24-week, multicenter, randomized, double-blind, placebo-controlled study with a 28-week extension. Diabetes Care. 2015;38(7):1218-27.

34. Wiviott SD, Raz I, Bonaca MP, Mosenzon O, Kato ET, Cahn A, et al. Dapagliflozin and cardiovascular outcomes in type 2 diabetes. N Engl J Med. 2019;380(4):347-57.

35. Verma S, Mazer CD, Yan AT, Mason T, Garg V, Teoh H, et al. Effect of empagliflozin on left ventricular mass in patients with type 2 diabetes mellitus and coronary artery disease: the EMPA-HEART cardiolink-6 randomized clinical trial. Circulation. 2019;140(21):1693-702.

36. Zinman B, Wanner C, Lachin JM, Fitchett D, Bluhmki E, Hantel S, et al. Empagliflozin, cardiovascular outcomes, and mortality in type 2 diabetes. N Engl J Med. 2015;373(22):2117-28.

37. Inagaki N, Kondo K, Yoshinari T, Maruyama N, Susuta Y, Kuki H. Efficacy and safety of canagliflozin in Japanese patients with type 2 diabetes: a randomized, double-blind, placebo-controlled, 12-week study. Diabetes Obes Metab. 2013;15(12):1136-45.

38. Leiter LA, Cefalu WT, de Bruin TW, Gause-Nilsson I, Sugg J, Parikh SJ. Dapagliflozin added to usual care in individuals with type 2 diabetes mellitus with preexisting cardiovascular disease: a 24-week, multicenter, randomized, double-blind, placebo-controlled study with a 28-week extension. J Am Geriatr Soc. 2014;62(7):1252-62.

39. Rosenstock J, Jelaska A, Zeller C, Kim G, Broedl UC, Woerle HJ. Impact of empagliflozin added on to basal insulin in type 2 diabetes inadequately controlled on basal insulin: a 78-week randomized, double-blind, placebo-controlled trial. Diabetes Obes Metab. 2015;17(10):936-48.

40. Kovacs CS, Seshiah V, Merker L, Christiansen AV, Roux F, Salsali A, et al. Empagliflozin as add-on therapy to pioglitazone with or without metformin in patients with type 2 diabetes mellitus. Clin Ther 2015;37(8):1773-88.e1.

41. Mathieu C, Ranetti AE, Li D, Ekholm E, Cook W, Hirshberg B, et al. Randomized, double-blind, phase 3 trial of triple therapy with dapagliflozin add-on to saxagliptin plus metformin in type 2 diabetes. Diabetes Care. 2015;38(11):2009-17.

42. Søfteland E, Meier JJ, Vangen B, Toorawa R, Maldonado-Lutomirsky M, Broedl UC. Empagliflozin as add-on therapy in patients with type 2 diabetes inadequately controlled with linagliptin and metformin: a 24-week randomized, double-blind. Parallel-Group Trial Diabetes Care. 2017:40(2):201-9.

43. Wilding JP, Woo V, Soler NG, Pahor A, Sugg J, Rohwedder K, et al. Longterm efficacy of dapagliflozin in patients with type 2 diabetes mellitus receiving high doses of insulin: a randomized trial. Ann Intern Med. 2012;156(6):405-15.

44. Yale JF, Bakris G, Cariou B, Nieto J, David-Neto E, Yue D, et al. Efficacy and safety of canagliflozin over 52 weeks in patients with type 2 diabetes mellitus and chronic kidney disease. Diabetes Obes Metab. 2014;16(10):1016-27.

45. Barnett AH, Mithal A, Manassie J, Jones R, Rattunde H, Woerle HJ, et al. Efficacy and safety of empagliflozin added to existing antidiabetes treatment in patients with type 2 diabetes and chronic kidney disease: a randomised, double-blind, placebo-controlled trial. Lancet Diabetes Endocrinol. 2014;2(5):369-84.

46. Grunberger G, Camp S, Johnson J, Huyck S, Terra SG, Mancuso JP, et al. Ertugliflozin in patients with stage 3 chronic kidney disease and type 2 diabetes mellitus: The VERTIS RENAL Randomized Study. Diabetes Ther. 2018;9(1):49-66.

47. Perkovic V, Jardine MJ, Neal B, Bompoint S, Heerspink HJL, Charytan DM, et al. Canagliflozin and renal outcomes in type 2 diabetes and nephropathy. N Engl J Med. 2019;380(24):2295-306.

48. Damman K, Beusekamp JC, Boorsma EM, Swart HP, Smilde TDJ, Elvan A, et al. Randomized, double-blind, placebo-controlled, multicentre pilot study on the effects of empagliflozin on clinical outcomes in patients with acute decompensated heart failure (EMPA-RESPONSE-AHF). Eur J Heart Fail. 2020;22(4):713-22.

49. McMurray JJV, Solomon SD, Inzucchi SE, Køber L, Kosiborod MN, Martinez $F A$, et al. Dapagliflozin in patients with heart failure and reduced ejection fraction. N Engl J Med. 2019;381(21):1995-2008.

50. Zelniker TA, Wiviott SD, Raz I, Im K, Goodrich EL, Bonaca MP, et al. SGLT2 inhibitors for primary and secondary prevention of cardiovascular and renal outcomes in type 2 diabetes: a systematic review and meta-analysis of cardiovascular outcome trials. Lancet. 2019;393(10166):31-9. 
51. Shao SC, Chang KC, Hung MJ, Yang NI, Chan YY, Chen HY, et al. Comparative risk evaluation for cardiovascular events associated with dapagliflozin vs. empagliflozin in real-world type 2 diabetes patients: a multi-institutional cohort study. Cardiovasc Diabetol. 2019;18(1):120.

52. Guo M, Ding J, Li J, Wang J, Zhang T, Liu C, et al. SGLT2 inhibitors and risk of stroke in patients with type 2 diabetes: a systematic review and metaanalysis. Diabetes Obes Metab. 2018;20(8):1977-82.

53. Shah SR, Luu SW, Calestino M, David J, Christopher B. Management of atrial fibrillation-flutter: uptodate guideline paper on the current evidence. J Community Hosp Intern Med Perspect. 2018;8(5):269-75.

54. Li WJ, Chen XQ, Xu LL, Li YQ, Luo BH. SGLT2 inhibitors and atrial fibrillation in type 2 diabetes: a systematic review with meta-analysis of 16 randomized controlled trials. Cardiovasc Diabetol. 2020;19(1):130.

55. Das SR, Everett BM, Birtcher KK, Brown JM, Januzzi JL Jr, Kalyani RR, et al. 2020 expert consensus decision pathway on novel therapies for cardiovascular risk reduction in patients with type 2 diabetes: a Report of the American College of Cardiology Solution Set Oversight Committee. J Am Coll Cardiol. 2020;76(9):1117-45

56. Shimizu W, Kubota Y, Hoshika Y, Mozawa K, Tara S, Tokita Y, et al. Effects of empagliflozin versus placebo on cardiac sympathetic activity in acute myocardial infarction patients with type 2 diabetes mellitus: the EMBODY trial. Cardiovasc Diabetol. 2020;19(1):148.

57. Chan YH, Lee HF, Li PR, Liu JR, Chao TF, Wu LS, et al. Effectiveness, safety, and major adverse limb events in atrial fibrillation patients with concomitant diabetes mellitus treated with non-vitamin K antagonist oral anticoagulants. Cardiovasc Diabetol. 2020;19(1):63.

58. Sardu C, Paolisso P, Ducceschi V, Santamaria M, Sacra C, Massetti M, et al. Cardiac resynchronization therapy and its effects in patients with type 2 DIAbetes mellitus OPTimized in automatic vs. echo guided approach. Data from the DIA-OPTA investigators. Cardiovasc Diabetol. 2020;19(1):202.

59. Tham YK, Bernardo BC, Ooi JY, Weeks KL, McMullen JR. Pathophysiology of cardiac hypertrophy and heart failure: signaling pathways and novel therapeutic targets. Arch Toxicol. 2015;89(9):1401-38.

60. Sano M. Hemodynamic effects of sodium-glucose cotransporter 2 inhibitors. J Clin Med Res. 2017;9(6):457-60.

61. Sano M. A new class of drugs for heart failure: SGLT2 inhibitors reduce sympathetic overactivity. J Cardiol. 2018;71(5):471-6.
62. Gronda E, Jessup M, lacoviello M, Palazzuoli A, Napoli C. Glucose metabolism in the kidney: neurohormonal activation and heart failure development. J Am Heart Assoc. 2020:9(23):e018889.

63. Han BG, Lee JY, Kim MR, Shin H, Kim JS, Yang JW, et al. Fluid overload is a determinant for cardiac structural and functional impairments in type 2 diabetes mellitus and chronic kidney disease stage 5 not undergoing dialysis. PLOS ONE. 2020;15(7):e0235640.

64. Hiramatsu T, Ito H, Okumura S, Asano Y, Iguchi D, Furuta S. Impact of glucagon like peptide-1 receptor agonist and sodium glucose cotransporter 2 inhibitors on type 2 diabetes patients with renal impairment. Diab Vasc Dis Res. 2020;17(6):1479164120971220.

65. Packer M, Anker SD, Butler J, Filippatos G, Zannad F. Effects of sodiumglucose cotransporter 2 inhibitors for the treatment of patients with heart failure: proposal of a novel mechanism of action. JAMA Cardiol. 2017;2(9):1025-9.

66. Bertero E, Prates Roma L, Ameri P, Maack C. Cardiac effects of SGLT2 inhibitors: the sodium hypothesis. Cardiovasc Res. 2018;114(1):12-8

67. Yurista SR, Silljé HHW, Rienstra M, de Boer RA, Westenbrink BD. Sodiumglucose co-transporter 2 inhibition as a mitochondrial therapy for atrial fibrillation in patients with diabetes? Cardiovasc Diabetol. 2020;19(1):5.

68. Shao Q, Meng L, Lee S, Tse G, Gong M, Zhang Z, et al. Empagliflozin, a sodium glucose co-transporter-2 inhibitor, alleviates atrial remodeling and improves mitochondrial function in high-fat diet/streptozotocininduced diabetic rats. Cardiovasc Diabetol. 2019;18(1):165.

69. Ferrannini E, Baldi S, Frascerra S, Astiarraga B, Heise T, Bizzotto R, et al. Shift to fatty substrate utilization in response to sodium-glucose cotransporter 2 inhibition in subjects without diabetes and patients with type 2 diabetes. Diabetes. 2016;65(5):1190-5.

70. Ferrannini E, Mark M, Mayoux E. CV protection in the EMPA-REG OUTCOME Trial: a "Thrifty Substrate" hypothesis. Diabetes Care. 2016:39(7):1108-14.

71. Barth AS, Tomaselli GF. Cardiac metabolism and arrhythmias. Circ Arrhythm Electrophysiol. 2009;2(3):327-35.

\section{Publisher's Note}

Springer Nature remains neutral with regard to jurisdictional claims in published maps and institutional affiliations.
Ready to submit your research? Choose BMC and benefit from:

- fast, convenient online submission

- thorough peer review by experienced researchers in your field

- rapid publication on acceptance

- support for research data, including large and complex data types

- gold Open Access which fosters wider collaboration and increased citations

- maximum visibility for your research: over 100M website views per year

At BMC, research is always in progress.

Learn more biomedcentral.com/submissions 